\title{
Pulmonary cryptococcosis mimicking solitary lung cancer in an immunocompetent patient
}

A 48-year-old Chinese male henhouse plain chest radiograph and CT scan keeper was admitted with dyspnoea. A indicated a solitary right lung mass with
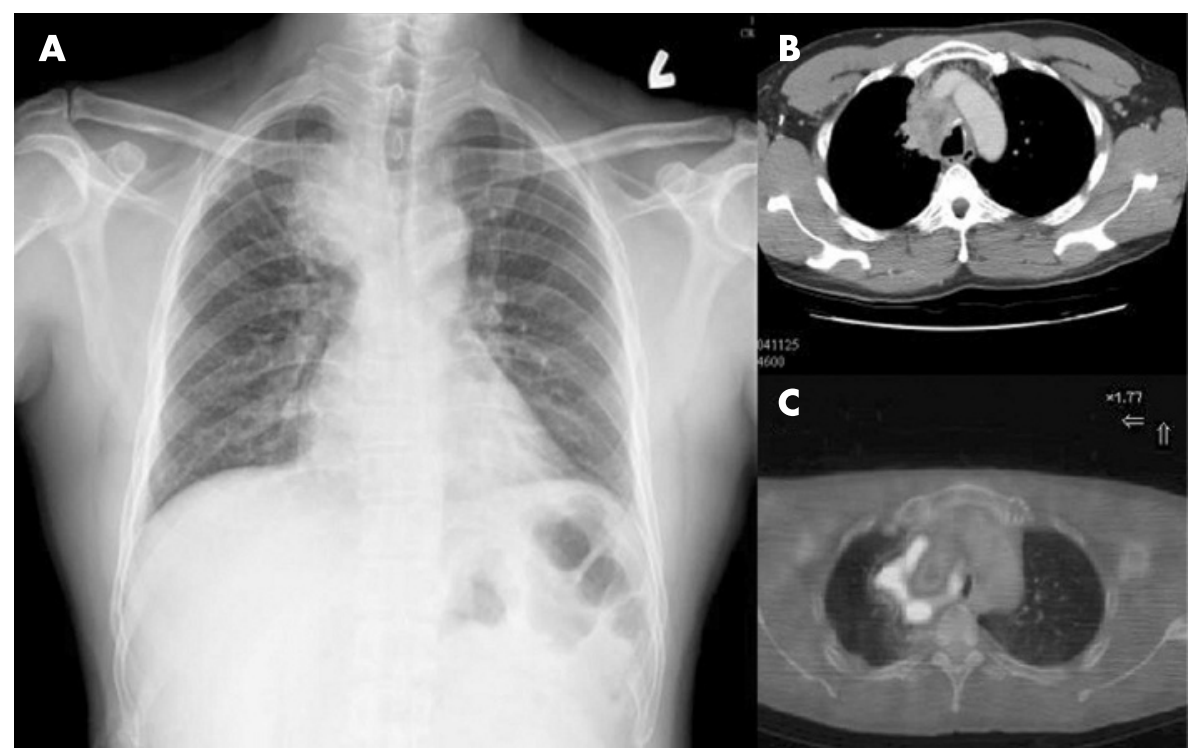

Figure 1 (A) Chest radiograph and (B) CT scan showing a solitary mass over the right upper lung area. (C) FDG-PET scan showing positive accumulation in the solitary mass.
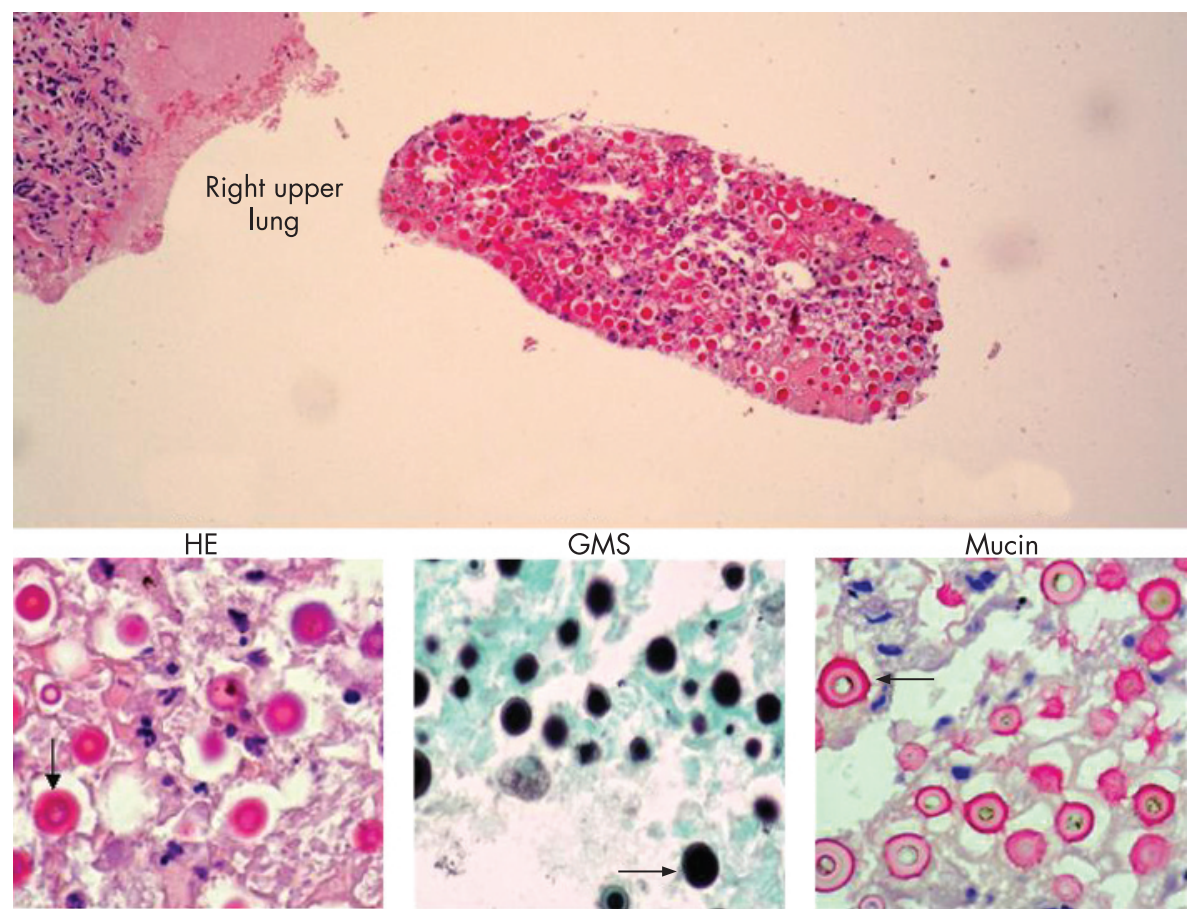

Figure 2 Microscopic examination of the lung tissue showing pulmonary cryptococcosis (arrow). $\mathrm{HE}$, haematoxylin-eosin stain; GMS, Gomori methenamine silver stain.

\section{Learning points}

- Pulmonary cryptococcus can present as a malignant mass with positive accumulation on the FDG-PET scan.

- Antifungal drugs may be an appropriate treatment without surgical intervention.

invasion to the trachea (fig $1 \mathrm{~A}$ and $\mathrm{B}$ ). Positron emission tomography with ${ }^{18} \mathrm{~F}$ fluoro-2-deoxy-D-glucose

(FDG-PET)

showed accumulation in the lung mass (fig 1C). A transbronchial biopsy specimen provided a pathological diagnosis of pulmonary cryptococcosis (fig 2). There were no malignant cells in the specimen. A fungus culture grew Cryptococcus neoformans, but a study of the cerebrospinal fluid showed no evidence of cryptococcal meningitis. A serum HIV test was negative. The patient was treated with oral itraconazole $400 \mathrm{mg} /$ day for 1 year without surgical intervention. A CT scan of the thorax after 2 years showed no evidence of the pulmonary lesion.

\section{DISCUSSION}

The high-resolution CT characteristics of pulmonary cryptococcosis in immunocompetent patients are multiple nodules and solitary nodules. ${ }^{1}$ FDG-PET is a relatively new imaging modality that facilitates the distinction between benign and malignant lesions, but some reports show accumulation on chronic inflammation. $^{2}$

\section{E-T Chang, ${ }^{1}$ A H Wang, ${ }^{3}$ C-B Lin, ${ }^{1}$ J-J Lee,} S-H Liü ${ }^{2}$

${ }^{1}$ Chest Medicine, Department of Internal Medicine, Buddhist Tzu Chi General Hospital, Hualien, Taiwan, ROC: ${ }^{2}$ Department of Nuclear Medicine, Buddhist Tzu Chi General Hospital, Hualien, Taiwan, ROC; ${ }^{3}$ Department of Bioengineering, School of Engineering and Applied Science, University of California-Los Angeles, Los Angeles, California, USA

Correspondence to: Dr C-B Lin, 707, Sec. 3 ChungYang Rd, Hualien, Taiwan 970, ROC; evan7622@yahoo. com.tw

\section{Competing interests: None.}

Patient consent: Patient consent was obtained to submit this case report.

Thorax 2008;63:478. doi:10.1136/thx.2007.079244

\section{REFERENCES}

Murayama S, Sakai S, Soeda H, et al. Pulmonary cryptococcosis in immunocompetent patients: HRCT characteristics. J Clin Imaging 2004;28:191-5.

2. Hsu CH, Lee CM, Wang FC, et al. F-18 fluorodeoxyglucose positron emission tomography in pulmonary cryptococcoma. Clin Nucl Med 2003:28:791-3. 